\title{
High stress monitoring of prestressing tendons in nuclear concrete vessels using fibre-optic sensors
}

\author{
M. Perry ${ }^{\mathrm{a} *}$, Z. Yan ${ }^{\mathrm{b}}$ Z. Sun ${ }^{\mathrm{b}}$ L. Zhang ${ }^{\mathrm{b}}$, P. Niewczas ${ }^{\mathrm{a}}$, M. Johnston \\ a Institute for Energy and Environment, University of Strathclyde, 204 George Street, Glasgow G1 1XW, United Kingdom \\ b Aston Institute of Photonic Technologies, Aston University, Birmingham B4 7ET, United Kingdom \\ c Civil Design Group, EDF Energy, Nuclear Generation, East Kilbride G74 5PG, United Kingdom
}

\begin{abstract}
Maintaining the structural health of prestressed concrete nuclear containments is a key element in ensuring nuclear reactors are capable of meeting their safety requirements. This paper discusses the attachment, fabrication and characterisation of optical fibre strain sensors suitable for the prestress mon- itoring of irradiated steel prestressing tendons. The all-metal fabrication and welding process allowed the instrumented strand to simultaneously monitor and apply stresses up to $1300 \mathrm{MPa}(80 \%$ of steel's ulti- mate tensile strength). There were no adverse effects to the strand's mechanical properties or integrity. After sensor relaxation through cyclic stress treatment, strain transfer between the optical fibre sen- sors and the strand remained at $69 \%$. The fibre strain sensors could also withstand the non-axial forces induced as the strand was deflected around a $4.5 \mathrm{~m}$ bend radius. Further development of this technology has the potential to augment current prestress monitoring practices, allowing distributed measurements of short- and long-term prestress losses in nuclear prestressed-concrete vessels.
\end{abstract}

\section{Introduction}

Prestressed concrete pressure and containment vessels (col- lectively referred to as PCVs in this paper) provide radiation and physical shielding for the reactor components they house. Pre- stressed concrete pressure vessels contain the reactor coolant fluid at the high operating pressures and temperatures experienced dur- ing operation, while containment vessels are designed to contain any release of radioactive material from the reactor circuit housed within the building in the event of a fault. These passive barriers are essential to a nuclear reactor's safety strategy, so the evolu- tion of their structural integrity has been the focus of decades of continuous monitoring and research (Ashar and Naus, 1983; Park, 2010). As concrete is weak under tension, PCVs can only func- tion effectively if their levels of compressive prestress, supplied by steel prestressing tendons, remains above a minimum design load. Ensuring this remains the case in the face of long-term pre-stress losses such as concrete creep, shrinkage and steel relaxation requires detailed measurements, obtained from prestress inspec- tion activities (Ghali, 1989; Anderson, 2005).

The prestress levels remaining in a PCV are inferred from continual assessments of tendon load, vessel dimensions and tem- perature. When tendon ducts are 'ungrouted', as is the case in the PCVs of UK nuclear power stations, tendon strands and wires can be mobilised after prestressing. This allows the average strand force to be measured directly via lift-off inspections or anchorage load cells (Smith, 1996). It is more common globally, however, for ducts to be grouted with cement in an effort to improve stress transfer between the tendons and vessel. This inhibits direct force measure- ments, so the prestress in grouted PCVs may be inferred from load cells installed on a small sample of ungrouted tendons in the struc- ture (USNRC, 1977). Some PCVs also contain vibrating wire strain gauges (VWSGs), which monitor linear strains in the vessel via the resonant frequency of a taut metal wire (Neild et al., 2005). Much like thermocouples, VWSGs are embedded into the PCV during con- 
struction and so cannot be accessed later for repair or replacement. This places stringent requirements on the sensors' reliability under continued radiation, temperature cycles and usage. This issue is especially relevant to existing generation II reactors which contain older sensing technologies (McFarlane et al., 1997).

There is currently a strong interest in the nuclear power gen- eration industry to augment prestress monitoring practices, to further improve the safety of existing and future reactors. The monitoring techniques above typically supply averaged measurements of tendon load with a $0.5 \mathrm{kN}$ force resolution (equivalent to a strain of 10 in 7-wire strand). However, immediate pre- stress losses, such as friction between the tendon and duct; and slippage at tendon anchorages, lead to tendon forces which vary as a function of distance along the duct within the vessel (Nawy, 2010). These spatially distributed tendon forces are not measured by existing techniques, and knowledge of them is important for building up an improved analysis of structural health in certain load cases.

Fibre optic sensors may provide a unique solution for auto- mated, distributed prestress monitoring, allowing the conversion of existing and future PCVs into smart structures (Udd, 1996). Fibre Bragg grating (FBG) sensors in particular, are wellsuited to non- invasive integration into linear systems, like prestressing tendon strands, due to their almost one-dimensional nature. Light weight, chemically inert, and immune to electromagnetic interference, FBG sensors detect changes in strain and temperature by measuring the modulated properties of light. As these point sensors can be seri- ally multiplexed, they can potentially form a highly cost-effective and extensible method for monitoring distributed strand strain. FBG strain sensor resolutions of 1 are common - indeed, some schemes with nanostrain resolutions have been proposed (Liu et al., 2010; Perry et al., 2012b). Fibre-instrumented strands may thus provide a feasible method for acquiring new prestress information with a high resolution.

In this paper, we present an all-metal process for fabricating and attaching FBG strain and temperature sensors to prestressing tendon strands. This allows us to demonstrate FBG sensors which can, for the first time, survive and monitor the strain in prestressing strands as they are stressed to $1300 \mathrm{MPa}(80 \%$ of the strand's ultimate tensile strength). Furthermore, careful selection of fibre and packaging materials means these sensors are suitable for use in nuclear environments. The proposed 'smart strand' technology is suited to both new build PCVs with grouted or ungrouted ducts and, due to the fact that the strands can be mobilised and replaced, exist- ing ungrouted PCVs. By verifying and augmenting current health monitoring practices, this technology may improve confidence and safety in not just current, but also future generations of nuclear power plants.

\section{Sensor design and fabrication}

\subsection{Fibre Bragg gratings}

A fibre Bragg grating (FBG) is a periodic modulation of the refrac- tive index of an optical fibre, as shown in Fig. 1. The modulation is typically $5-20 \mathrm{~mm}$ long and is written by side illuminating a photosensitive fibre with an ultraviolet laser. 


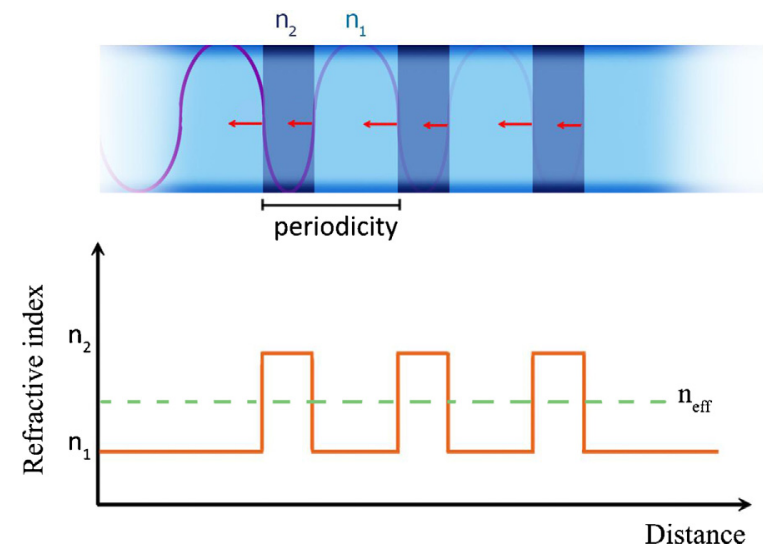

Fig. 1. Diagram showing an FBG and its refractive index modulation. Thousands of modulations are typical, but only three are shown here for clarity.

When broadband light, guided within the fibre, meets the index modulation, a narrow set of wavelengths are reflected back towards the light source (Hill and Meltz, 1997). The central wavelength reflected is denoted the Bragg peak, B, and it is a function of the refractive index and periodicity of the grating. Altering the strain, $\varepsilon$, or temperature, T, applied to the FBG affects these parameters. This leads to fractional shifts in the Bragg peak described by:

$$
\frac{\Delta \lambda_{B}}{\lambda_{B}}=K_{\varepsilon} \varepsilon+K_{T} T
$$

where $\mathrm{K} \varepsilon$ and $\mathrm{KT}$ are the strain and temperature sensitivity of the FBG, respectively. To obtain strain measurements independently of temperature, a second nearby reference FBG is introduced. This reference is isolated from strain, allowing temperature effects to be measured independently and then subtracted from the strain sen- sor. This referencing principle holds for other extraneous variables which affect $\mathrm{B}$, such as radiation and pressure.

The initial Bragg wavelength of the FBG can be customised during the writing process. A serial array of FBGs, can thus be man- ufactured to reflect a series of different Bragg wavelengths. This allows serial multiplexing and reading of a chain of distributed strain and reference sensors using a single light source and inter- rogation device, as shown in Fig. 2.

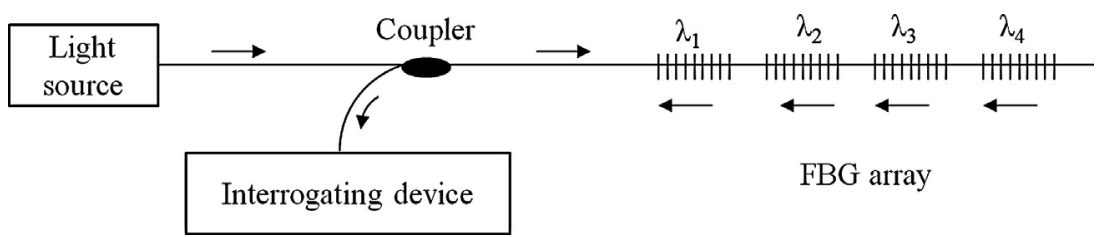

Fig. 2. Fibre diagram showing the principle of serial wavelength division multiplexing.

\subsection{Packaging of FBGs}

Due to the fundamental weaknesses of glass, encapsulation of the FBG sensor is crucial in ensuring long-term mechanical, ther- mal and radiation reliability. A problem that frequently impedes the fibre-instrumentation of metal parts is that of achieving a strong, reliable glass-to-metal bond that does not mechanically or thermally damage either component. This is essential in the current application, as prestressing strands are placed under enor- mous $1300 \mathrm{MPa}$ stresses, corresponding to $80 \%$ of the ultimate tensile strength (UTS) of steel. A common method of 
circumvent- ing the glass-to-metal bonding problem in strand instrumentation is to avoid using steel strands altogether (Li et al., 2004b). Optical fibres can be more conveniently embedded or glued to fibre- reinforced plastic (FRP) prestressing strands, due to the material's lower hardness (Zhou and Sim, 2002). Unfortunately, the weak shear strength of FRPs and their enhanced degradation under irradiation, moisture, temperature and acidity make them less suited to the PCV environment (Chin et al., 1997). FRP strands also display different mechanical, thermal and relaxation proper- ties, making them unrepresentative of the existing steel strands in PCVs.

Given that steel strands must be used, another obvious glass bonding solution is epoxy. This is indeed a simple, room- temperature method, but the limited mechanical strength of epoxies means attached sensors tend to fail before the $80 \%$ UTS threshold is reached ( $\mathrm{Li}$ et al., 2004a). Mechanical creep is also a major problem once epoxies are stressed to beyond 50\% of their tensile strength (Majda and Skrodzewicz, 2009). This significantly reduces a bonded strain sensor's accuracy. Most epoxies are also degraded by radiation, and while novel radiation-resistant epoxies have been developed, these display unacceptably low mechanical strengths (Prokopec et al., 2009).

To overcome the limitations of these conventional bonding methods, we have developed an all-metal encapsulation and attachment process for FBG sensors. The exclusive use of metal joining techniques helps to provide the thermal, mechanical and radiation resistance and diminished creep essential for long-term prestress monitoring (Beranek et al., 2001; Mei and Morris, 1992). In previous work, we characterised a high-temperature induction brazing process for encapsulating FBGs in metallic capillaries, with- out lasting thermal damage to the fibre (Perry et al., 2013). The design of the packaged and welded FBG is shown in Fig. 3. As shown, the FBG is brazed into a capillary for mechanical protection under bending, tension and compression. Careful selection of the FBGs' glass and coating chemistry were used to ensure their long-term survivability under continued neutron-gamma radiation (Perry et al., 2012a; Gusarov and Hoeffgen, 2013), high temperatures (Canning, 2012) and large mechanical stresses (Kobrin, 1997). Due care was also given to the fibre's treatment and handling through- out the packaging process and this allowed the FBGs to retain the high tensile strength demanded by the application (Olshanksy and Maruer, 1976).

While brazing is suitable for packaging fibre sensors, expos- ing prestressing strands to high-temperatures can lead to strength reduction and de-hardening of the steel. Furthermore, rapid cooling can lead to martensite formation, causing the steel to become brittle and weak. To minimise strand damage, the packaged FBG sen- sors were attached using an electrical resistance spot welder. This highly localised welding method reduces the volumes of marten- sitic growth, which in turn minimises adverse thermal effects to the steel's mechanical properties. FBG strain sensors were welded at both ends to allow strain to be transferred from the strands to the FBG. Reference FBGs were welded at one end only, so that they could independently measure and correct for fluctuations in ambient temperature. 


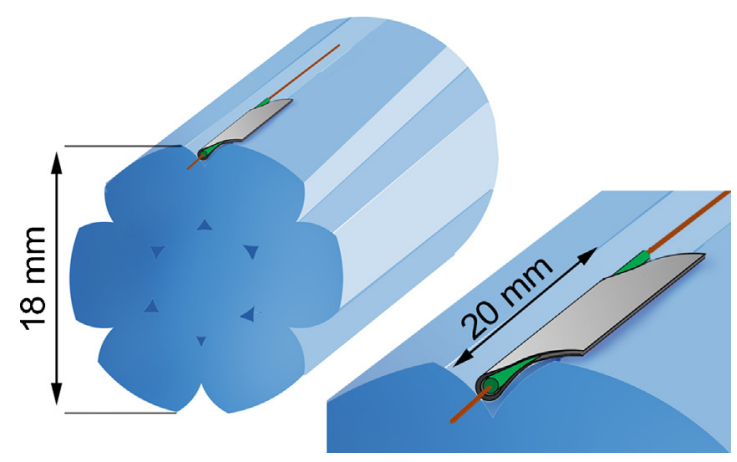

Fig. 3. Diagram of a capillary-encapsulated fibre Bragg grating (FBG) attached to a prestressing strand

\section{Smart strand characterisation and results}

\subsection{Prestressing strand damage}

To empirically verify the effects of spot-welding on a strand's properties, ten sensors were spot-welded to each of two strands, T1 and T2, while two control strands, T3 and T4, were left untreated. The strands were all placed under destructive tensile strength, metallographic and micro-hardness tests. As shown in Fig. 4, ten- sile tests until fracture revealed that the yield and ultimate tensile strengths of all strands were higher than the values specified in British Standard BS 5896:1980. The modulus of elasticity of all spec- imens was $185 \mathrm{GPa}$, regardless of whether or not they were exposed to spot-welding.

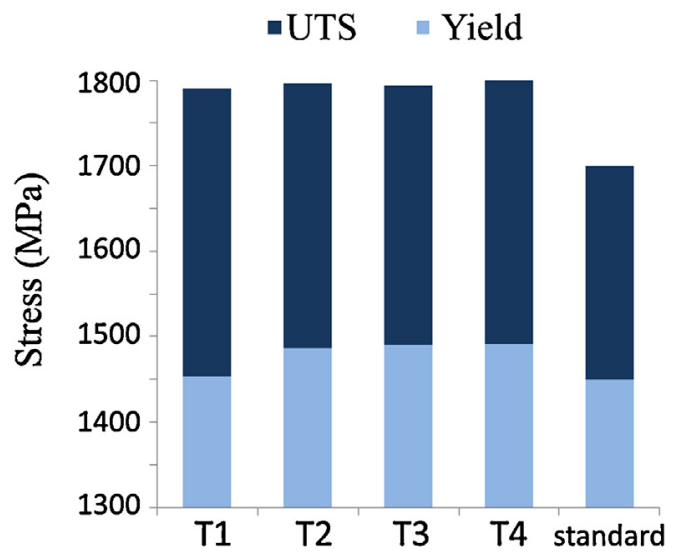

Fig. 4. Comparison of ultimate tensile and yield strength of spot-welded (T1 and T2) and untreated (T3 and T4) strand specimens.

The mechanical hardness of both welded and non-welded regions of the strands was tested using Vickers micro-indentation under $300 \mathrm{~g}$ force. The $914 \mathrm{HV}$ average hardness valuefound in welded regions was almost double that of the unwelded regions at $481 \mathrm{HV}$. This high hardness value demonstrates that martensitic transformations arose at weld locations due to rapid cooling. To gauge the extent of the martensite formation, cross-sections from spot-welded regions of the strand were cut, polished and etched to reveal the crystal microstructure. A typical optical microscope image is shown in Fig. 5. The images reveal that the martensitic transformations from spot-welding only penetrate approximately $0.1 \mathrm{~mm}$ from the strand surface. The strand's mechanical properties were unaffected because of this minimal damage. 


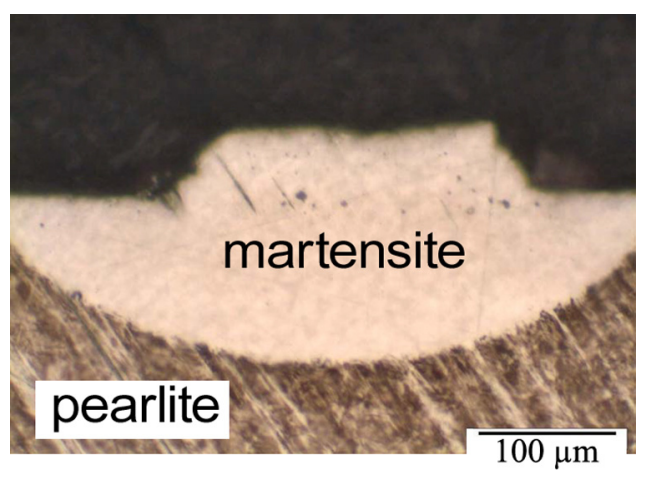

Fig. 5. Microscope image of spot-welded region revealing martensite formation.

\subsection{Temperature characterisation}

Because the coefficient of thermal expansion of glass fibre is low, the temperature sensitivity of unbonded, reference FBGs is mainly dependent on thermally induced refractive index changes. The thermal sensitivity of the welded FBG strain sensor, on the other hand, is heavily influenced by the thermal expansion of the steel strand to which it is attached.

The wavelength shifts in unbonded reference and welded sensor FBGs between 25 and $55 \circ \mathrm{C}$ are shown in Fig. 6 . The welded FBG has a temperature sensitivity which is some $20 \%$ higher due to the influ- ence of the strand's thermal expansion. This calibration graph for temperature allows future strain measurements to be made independently of thermal effects. It also allows the reference FBG to act independently as a thermometer, essentially replacing the role of a thermocouple. This could potentially augment the coverage of the thermocouples placed in the PCV during construction.

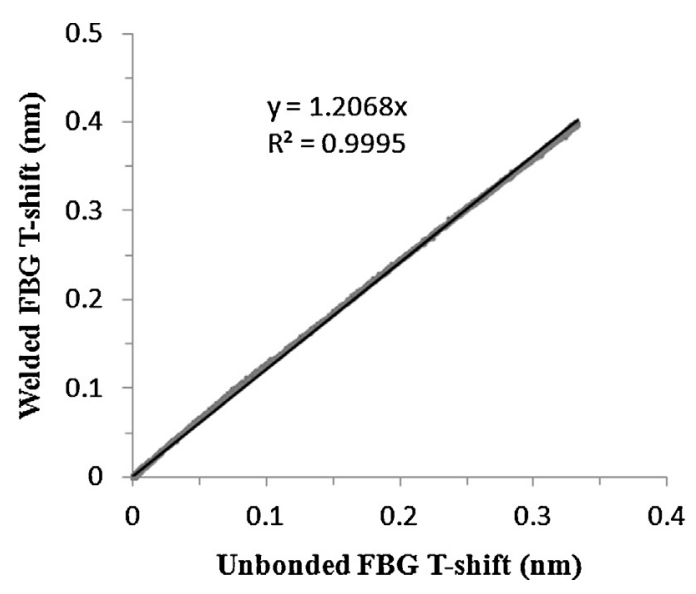

Fig. 6. Comparison of temperature-induced Bragg wavelength shifts (in nanome- ters, $\mathrm{nm}$ ) in unbonded and welded FBGs.

\subsection{Strain transfer coefficient and bending}

The strain transfer coefficient is defined by the ratio of the strain applied to the strand to that measured in the fibre sensor. A can- tilever loading rig was used to apply small strains to a $1 \mathrm{~m}$ long prestressing strand sample. The strand was fixed at one end while bending forces up to $25 \mathrm{~N}$ were applied to the free end. Spot-welded and epoxied FBG strain sensors were bonded to the top surface of the strand cantilever, 3 $\mathrm{cm}$ from the fixed point. This allowed the strain transfer of welded FBG sensors to be compared to those bonded using the conventional method of epoxy. The strain measured by both sensors as a function of end force is shown in Fig. 7. 
While both sensors had a linear response as expected, the strain transfer of the allmetal welded FBG was only $87 \%$ that of the epox- ied sensor. This is because strain transfer is highly dependent on the contact area between the prestressing strand and the FBG (Li et al., 2009). Epoxying maximises this contact area (and hence the strain transfer) as it envelops the FBG, while welding is a highly direc- tional and localised process (Wei et al., 2001). Nevertheless, the cantilever experiment does demonstrate that the welded FBG sen- sors are able to withstand and measure non-axial stresses. The $25 \mathrm{~N}$ end force is equivalent to a bending radius of approximately $4.5 \mathrm{~m}$ - several times less than the radius of the tendon duct curvature in a typical PCV.

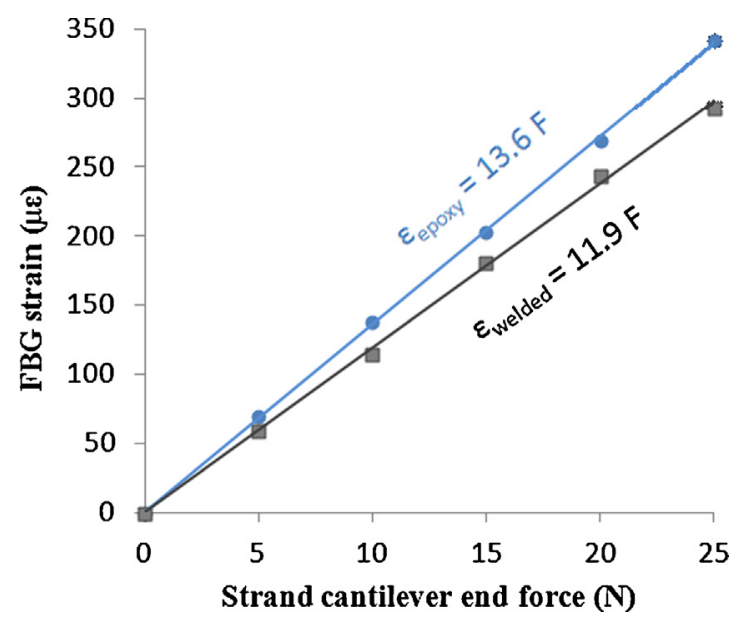

Fig. 7. Strain response of welded and epoxied FBG sensors in a cantilever set up.

\subsection{Relaxationstudies}

The response of the spot-welded FBG strain sensors was charac- terised under high stress by loading a $1 \mathrm{~m}$ long instrumented strand in mechanical tensile testing machine. The smart strand was de- stressed, then stressed to $1300 \mathrm{MPa}$ and held for 4 min. This process was repeated four times in total. Fig. 8 shows the decrease in strain measured by the FBG sensors during the holding phase. The final strain losses for the first, second and third stressing operations were $12 \%, 4 \%, 3 \%$ and $0.5 \%$, respectively. As the strands are stress relieved during manufacture, this relaxation is likely occurring principally in the FBG sensor packaging attachment as a consequence of the thermal stresses introduced during fabrication.

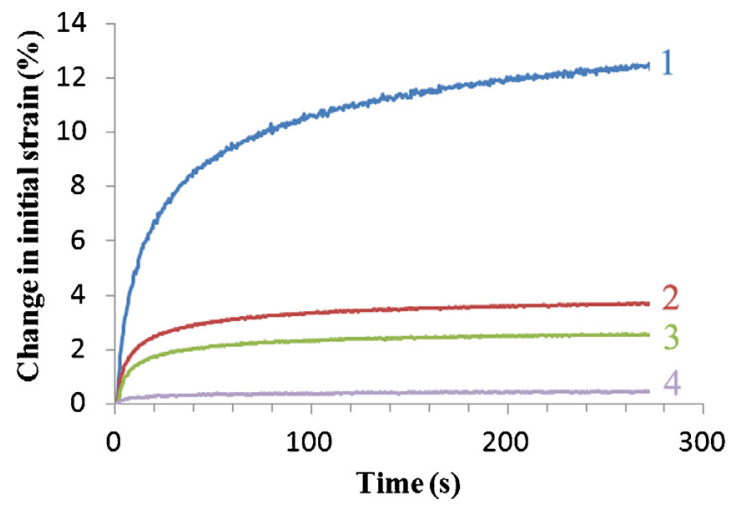

Fig.8. Stress relaxation of smart strand during four subsequent stressing operations to 1300 MPa.

Upon a fifth stressing operation, the holding time was extended to $10 \mathrm{~h}$, resulting in a total relaxation of $0.6 \%$. As shown in Fig. 9 the strain loss can be parameterised by 
logarithmic and power laws. This parameterisation is typical of steel relaxation and agrees with previous studies (Ghali and Trevino, 1985).

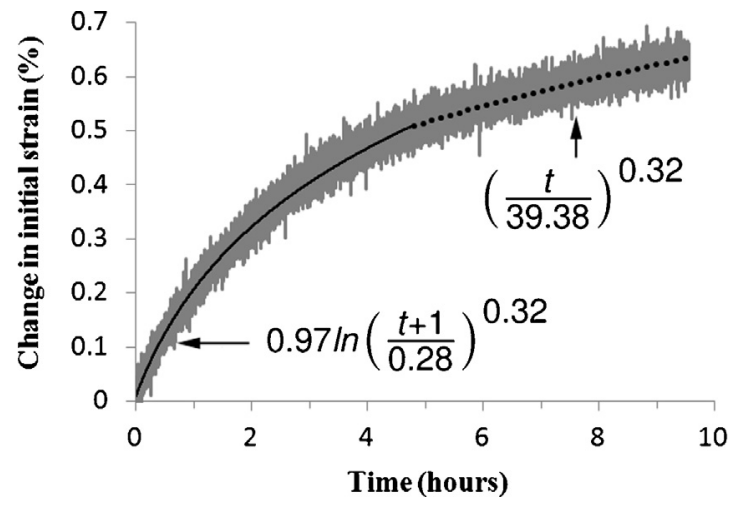

Fig.9. Parameterisation of the stress relaxation of sensors during $10 \mathrm{~h}$ of holding at $1300 \mathrm{MPa}$.

3.5. Linear high tensile strain measurements

Once relaxation was deliberately induced through cyclic load- ing, stress in the metrelong strand could be measured more reliably. The strain measured by the FBG strain sensor was mea- sured as strand stress was increased incrementally in five steps up to $1300 \mathrm{MPa}$. The linear relationship between these variables, shown in Fig. 10, suggests that strain transfer between the strand and sensor after relaxation was around 69\%. Fig. 10 was used as a calibration curve for subsequent stressing operations, allowing the FBG to act as a stress sensor in real time. As shown in Fig. 11, the welded strain sensor was capable of measuring prestressing strand stress as it was cycled up to $1300 \mathrm{MPa}$, or $80 \%$ UTS.

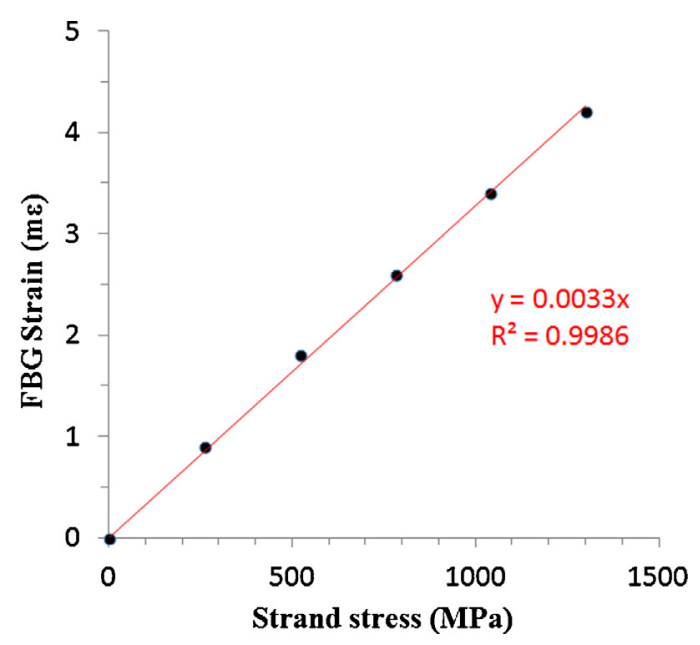

Fig. 10. Strain response of welded FBG sensor under strand stress up to $1300 \mathrm{MPa}$. 


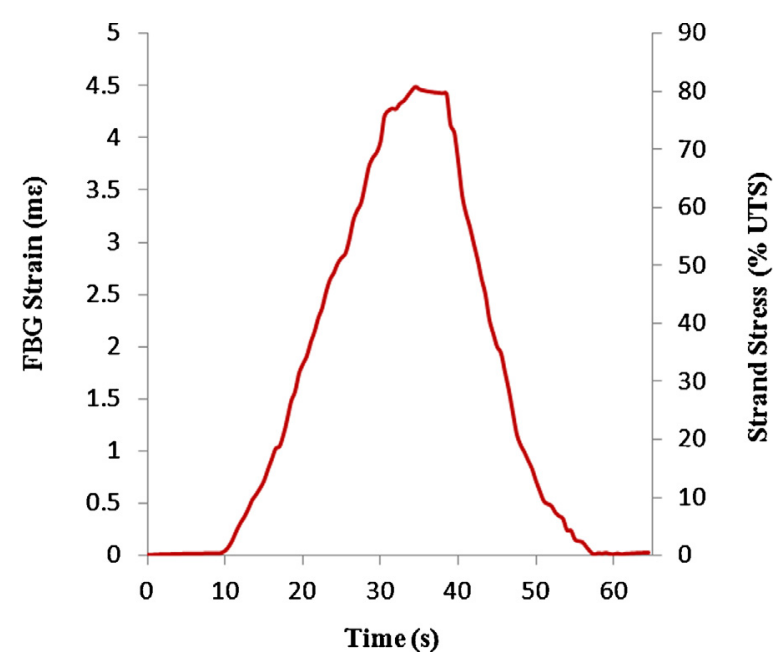

Fig. 11. Calibrated FBG strain sensor measuring stress as strand cycled between $0 \%$ and $80 \%$ UTS.

\section{Discussion and future work}

The work here has shown that radiation-resistant FBG strain sensors can survive and measure the stress in prestressing strands up to $80 \%$ UTS. Crucially, the attachment of the sensors does not affect strand integrity. This means that conventional steel prestressing strands can be used as a sensing medium - allowing the strands to uphold structural health while simultaneously moni- toring it. The strain transfer between the strand and sensors was around 69\%, slightly reduced due to relaxation and the directional nature of spot-welding. Lower strain transfer values may in fact be beneficial as they limit the strain applied to the FBGs, reducing the chances of fibre failure.

Due to the modular nature of the FBG sensors presented here, scaling the proposed smart strand technology up to real PCV pre- stress monitoring applications may be relatively straightforward and cost-effective. Sensors could, in future, be attached during or before strand replacement activities, from a reel of prepared fibre sensors. A combination of wavelength division multiplexing and parallel fibre lines will allow a single optical interrogator to monitor numerous strands within the PCV with a high level of redundancy. This redundancy may be essential, as monitoring strand stress inde- pendently of bend and wire slippage will require multiple strain sensors.

The results from lab demonstrations presented here encourage further investigation. Before field tests in model structures are con- ducted, further studies are required to characterise the dependence of relaxation on the materials and processing of the FBG strain sen- sor. Cyclic stressing of the sensor packaging before attachment may reduce relaxation, allowing the smart strand to begin functioning immediately upon its initial prestressing. Relaxation may also be enhanced under radiation, so long-term irradiation studies will be required to empirically prove that the proposed smart strand can measure stress reliably in PCVs, though most tendons experience minimal radiation doses. Characterisation of the strain measured at different ambient temperatures may also be required as FBG strain sensitivity can be temperature dependent (O'Dwyer et al., 2004). Finally, making sensor fabrication and attachment methods automated will allow the strain and temperature sensitivity to be predicted and characterised more reliably, enhancing the system's performance in industrial applications.

While the strain transfer of the spot-welded sensors is slightly less than that of 
epoxies, the final adopted technique has demon- strated sensors with a much higher strain range. Obtaining these benefits requires more intensive manufacturing processes, but the fact that the fibre sensors can continue to withstand and mea- sure high strains after processing is an indication that the high temperature processing has not caused adverse thermal damage. Furthermore, as the sensors' packaging and bonding mechanisms are completely metallic, they are less likely to be susceptible to radiation damage, but future work will be required to prove this explicitly.

There are other aspects of this technology which will require addressing before the smart strand can be fully commercialised. For example, there is potential for mechanical damage of the glass fibres during their installation into real structures as a consequence of mishandling, misalignment, pinching or bending, particularly at the tendon anchorages as they are inserted into the PCV. These issues will require detailed consideration of current tendon instal- lation procedures and perhaps design changes to allow the fibres to meet the physical demands of installation.

Note that while the smart strand may be a viable method of measuring the majority of strain-inducing prestress losses, it can- not measure those losses which do not induce strain, such as strand relaxation. As strand relaxation is a relatively large effect in new strands, it can be measured with conventional methods, such as load cells at the anchorages. Distributed strains measured by the FBG sensors could then be combined with force measurements from load cells or lift-off inspections to provide crossverification and reduce the probability of common mode failures of monitor- ing instrumentation. A comparison of anchorage force and strand strain profiles may also provide some clues as to the effective stiff- ness of strands. This can be used as an indicator of a strand's friction and corrosion state.

5. Conclusions

Radiation resistant optical fibre strain sensors were bonded to steel prestressing strands using an all-metal packaging and attach- ment process. Resistance spotwelding of the sensors to the strand was found to lead to a localised martensitic phase transformation which penetrated only $0.1 \mathrm{~mm}$ from the strand surface. As dam- age was minimal, spot-welding was found to have no effect on the mechanical properties of the strand.

The welded FBG strain sensors displayed relaxation upon initial stressing due to residual thermal stresses introduced during fab- rication. Once relaxation was deliberately induced through stress cycling, the strain transfer between the prestressing strand and sensors remained at $69 \%$. After relaxation, the fibre strain sensors were able to reliably measure strand stresses up to $1300 \mathrm{MPa}$. Cantilever experiments also revealed the sensors could withstand non-axial stress as the strand was deflected around a $4.5 \mathrm{~m}$ bend radius. Sensible extensions of the technology developed here could allow distributed measurements of short- and long-term prestress losses in the concrete vessels surrounding nuclear reactors. Such a scheme would reduce the probability of common mode failures for structural monitoring instrumentation and methods, and further ensure the continued safe operation of nuclear power plants in this generation and the next.

\section{Acknowledgements}

This work was supported by the Engineering and Physical Sciences Research Council (EPSRC) and EDF Energy Nuclear Gen- eration, Ltd.

\section{References}

Anderson, P., 2005. Thirty years of measured prestress at Swedish nuclear reactor 
containments. Nuclear Engineering and Design 235, 2323-2336.

Ashar, H., Naus, D.J., 1983. Overview of the use of prestressed concrete in U.S. nuclear power plants. Nuclear Engineering and Design 75, 425-437.

Beranek, M.W., Rassaian, M., Tang, C.-H., St John, C.L., Loebs, V.a., 2001. Characterization of $63 \mathrm{Sn} 37 \mathrm{~Pb}$ and $80 \mathrm{Au} 20 \mathrm{Sn}$ solder sealed optical fiber feedthroughs subjected to repetitive thermal cycling. IEEE Transactions on Advanced Packag- ing 24, 576-585.

Canning, J., 2012. Regenerated gratings for optical sensing in harsh environments. In: Advanced Photonics Congress. OSA, Washington, DC, pp. BTu3E.3.

Chin, J., Aouadi, K., Nguyen, T., 1997. Effects of environmental exposure on fiberreinforced plastic (FRP) materials used in construction. Journal of Composites Technology and Research 19, 205-213.

Ghali, A., 1989. Stress and strain analysis in prestressed concrete: a critical review. PCI Journal 31, 80-97.

Ghali, A., Trevino, J., 1985. Relaxation of steel in prestressed concrete. Journal of Prestressed Concrete Institute 30, 82-90.

Gusarov, A., Hoeffgen, S.K., 2013. Radiation effects on fiber gratings. IEEE Transac- tions on Nuclear Science 60, 2037-2053.

Hill, K.O., Meltz, G., 1997. Fiber Bragg grating technology fundamentals and overview. Journal of Lightwave Technology 15, 1263-1276.

Kobrin, B., 1997. Fibre strength unaffected by on-line writing of single-pulse Bragg gratings. Electronics Letters 33, 1333-1334.

Li, E., Xi, J., Chicharo, J.F., Liu, T., Li, X., Jiang, J., Li, L., Wang, Y., Zhang, Y., 2004a. The experimental evaluation of FBG sensor for strain measurement of prestressed steel strand. Proceedings of SPIE, Smart Structures, Devices, and Systems II 5649, 463-469.

Li, H., Zhou, G., Ren, L., Li, D., 2009. Strain transfer coefficient analyses for embedded fiber bragg grating sensors in different host materials. Journal of Engineering Mechanics 135, $1343-1353$.

Li, H.-N., Li, D.-S., Song, G.-B., 2004b. Recent applications of fiber optic sen- sors to health monitoring in civil engineering. Engineering Structures 26, 1647-1657.

Liu, Q., He, Z., Tokunaga, T., Hotate, K., 2010. An ultra-high-resolution FBG static- strain sensor for geophysics applications. In: Fourth European Workshop on Optical Fibre Sensors.

Majda, P., Skrodzewicz, J., 2009. A modified creep model of epoxy adhesive at ambient temperature. International Journal of Adhesion and Adhesives 29, 396-404.

McFarlane, J., Smith, L., Baird, C., 1997. In: Byars, E., McNulty, T. (Eds.), Management of Concrete Structures for Long-Term Serviceability: The Inspection and Evaluation of Nuclear Related Civil Engineering Structures. Thomas Telford, Sheffield, UK, pp. 85-86.

Mei, Z., Morris, J., 1992. Superplastic creep of low melting point solder joints. Journal of Electronic Materials 21.

Nawy, E., 2010. Prestressed Concrete: A Fundamental Approach, fifth ed. Prentice Hall, New Jersey, US.

Neild, S.a., Williams, M.S., McFadden, P.D., 2005. Development of a vibrating wire strain gauge for measuring small strains in concrete beams. Strain 41, 3-9. O'Dwyer, M., Ye, C.-C., James, S., Tatam, R., 2004. Thermal dependence of the strain

response of optical fibre Bragg gratings. Measurement Science and Technology

15, 1607-1613. Olshanksy, R., Maruer, R., 1976. Tensile strength and fatigue of optical fibers. Journal

of Applied Physics 47, 4497-4499. Park, J., 2010. Technical requirements and applications for ISI of prestressed concrete

nuclear containments with ungrouted tendons. Nuclear Engineering and Design

240, 3682-3686. Perry, M., Niewczas, P., Johnston, M., 2012a. Effects of neutron-gamma radiation on

fiber Bragg grating sensors: a review. IEEE Sensors Journal 12, 3248-3257.

Perry, M., Niewczas, P., Johnston, M., Cook, K., Canning, J., Bragg, A.F., Fbg, G., 2013.

Induction brazing of type-I fiber Bragg gratings into Kovar ferrules exploiting 
Curie transition. IEEE Sensors Journal 13, 816-823. Perry, M., Orr, P., Niewczas, P., Johnston, M., 2012b. Nanoscale resolution interro-

gation scheme for simultaneous static and dynamic fiber Bragg grating strain

sensing. Journal of Lightwave Technology 30, 3252-3258. Prokopec, R., Humer, K., Maix, R.K., Fillunger, H., Weber, H.W., 2009. Influence of

various catalysts on the radiation resistance and the mechanical properties of cyanate ester/epoxy insulation systems. Fusion Engineering and Design 84, 1544-1547.

Smith, L.M., 1996. In-service monitoring of nuclear-safety-related structures. The Structural Engineer 74, 210-211.

Udd, E., 1996. Fiber optic smart structures. Proceedings of the IEEE 84, 1898-1918. USNRC, 1977. US Nuclear Regulatory Commission: Office of Standards Develop- ment: Inservice Inspection of Prestressed Concrete Containment Structures with

Grouted Tendons. RG 1.90 Revision 1. Wei, C.Y., Ye, C.C., James, S.W., Tatam, R.P., Irving, P.E., 2001. An experimental

approach to quantify strain transfer efficiency of fibre Bragg grating sensors to host structures. In: The 13th International Conference on Composite Materials, ICCM-13, Beijing, China.

Zhou, G., Sim, L., 2002. Damage detection and assessment in fibre-reinforced com- posite structures with embedded fibre optic sensors - review. Smart Materials and Structures 11, 925-939. 